improve patients' experience by encouraging early intervention for best symptom management.

Methods An audit of referrals was conducted during 22/05/ 2017 to 26/07/2017. This was achieved by logging all referrals, locality, referrer name and designation. This was to map what GP practices, community/secondary services were referring and identify gaps in areas across the city to ascertain engagement and training needs.

A survey was undertaken through SurveyMonkey to measure stakeholders' knowledge of the service, time limit 15/08/ 2017 - 30/08/2017. Five questions were identified, the name of the organisation was requested and job role but anonymity was respected.

Outcome From the audit 92 referrals were received during the time period from a combination of primary and secondary services clearly showing gaps in areas across the city. 200 surveys were sent out and 65 responses received. Key findings were:

- $98 \%$ of people had heard of Compton Lymphoedema Care but knowledge and understanding of the services offered differed

- $28 \%$ of professionals weren't aware of the support group

- $25 \%$ were unsure how or who could refer.

Conclusion The project was successful in identifying a snapshot of demographic data across the city that has clearly identified gaps. Furthermore, the survey has identified a need for increased community engagement, improved communication, marketing strategies and education. Early intervention supports best patient management and reduces risks associated with long term swelling (British Lymphoedema Society, 2010).

\section{P-96 TO LOCALLY IMPLEMENT A LYMPHOEDEMA AND HOSPICE AWARENESS EDUCATION PROGRAMME}

Lynda Parrish, Katherine Newman. Ashgate Hospicecare, Chesterfield, UK

10.1136/bmjspcare-2018-hospiceabs. 121

Background A dedicated lymphoedema specialist clinic in a hospice setting has provided lymphoedema treatment for more than 14 years for people living with a cancer diagnosis (PLWCD). Despite this, many people referred to the service were not aware of lymphoedema, the risk factors or the role of Hospicecare.

Aims To implement an education programme. To provide knowledge and skills through an education programme for healthcare professionals (HCPs) to identify lymphoedema and how to refer to the service. To empower PLWCD on how to reduce the risks of developing lymphoedema and to recognise when to seek early intervention and a referral to the clinic. To demonstrate how hospice and palliative care services are changing and adapting.

Method In addition to formal training days, and an acknowledgement that 'one size doesn't fit all', alternative education sessions were incorporated within nurse leadership programmes, GP training and Primary Healthcare trust lunchtime sessions. Also education for cancer support groups and post cancer treatment 'Moving forward groups'.

Results Since March 2017, a total of 260 HCPs, 40 students and more than 100 PLWCD have received education and are now better informed about lymphoedema. Changing the concept of the role of the hospice.
Conclusion Responding to overwhelming positive feedback and recommendations received, all previous education sessions for HCPs will continue with additional specific sessions for other disciplines. An evening seminar will also be introduced. One awareness session for PLWCD has resulted in four appropriate referrals to the service for treatment, who without this education programme would not now be receiving specialist care.

\section{P-97 LEANING IN - MENTAL ILLNESS, PALLIATIVE AND END OF LIFE CARE - THE VIEWS OF PATIENTS AND CARERS}

\footnotetext{
1,2,3 Jed Jerwood, 'Jane Coad, 'Gillian Ward, ${ }^{1}$ Nikki Holliday, 'Diane Phimister. 'Coventry University, Coventry, UK; ${ }^{2}$ Birmingham and Solihull Mental Health Foundation Trust, Birmingham, UK; ${ }^{3}$ John Taylor Hospice, Birmingham, UK
}

10.1136/bmjspcare-2018-hospiceabs. 122

Background Research which concerns the experiences and expectations of people with mental illnesses and terminal conditions is extremely limited both in the UK and internationally. What is known is that people with mental ill health experience higher rates of many physical health issues, later diagnosis and poorer prognosis than the general population (Royal College of Psychiatrists, 2013). People with mental illness experience many barriers when trying to access palliative and end of life care services and are poorly supported following terminal diagnosis by mental health services (Jerwood, Phimister, Ward et al., 2018).

Aim The aim of this research was to conduct interviews with patients with histories of mental illness and terminal physical conditions, and their carers, to better understand their experiences and expectations of palliative and end of life care, and how more effective support and care could be provided by palliative, end of life and mental health care services.

Method Eight semi-structured interviews were carried out with patients and carers. Interviews were transcribed and a thematic analysis (Braun \& Clarke, 2006) carried out. Key themes were drawn from the interview data. These themes were used to inform a co-design process to develop a resource to improve care.

Findings This is the first research study which explores the views of patients (and their carers) with mental illnesses and terminal conditions about their end of life care needs, experiences and expectations. Key themes from the interviews will be presented with implications for clinical practice explored.

NB - The findings are being written up at the time of submitting this abstract so this will be completed work by the time of the conference.

\section{P-98 REVIEW OF THE PALLIATIVE CARE NEEDS OF PEOPLE WITH MOTOR NEURONE DISEASE AND INFORMAL CAREGIVERS}

1,2Victoria Turner, ${ }^{2}$ Kate Flemming. 'Leeds Teaching Hospitals NHS Trust, Leeds, UK; ${ }^{2}$ University of York, York, UK

10.1136/bmjspcare-2018-hospiceabs. 123

Introduction Despite being a terminal neurodegenerative disease, the role of palliative care is less recognised for motor neurone disease (MND) than for conditions such as cancer. The palliative care needs of patients and carers must be understood in order to best configure policy and health care services for this population. 
Aim To explore the palliative care needs of adult patients and their informal carers living with MND, or bereaved carers of people with $\mathrm{MND}$, through a systematic review of qualitative research.

Method Searches were conducted in four electronic databases (MEDLINE, CINAHL, PsycINFO, Social Science Citation Index) using terms for MND, ALS, palliative care, and a qualitative research filter. Relevant data were extracted from included papers, which were also appraised for quality. The review was conducted using Thematic Synthesis, with the papers coded in order of disease trajectory.

Results 410 papers were identified and 39 included, representing the experiences of 456 people with MND and 345 informal caregivers.

Two sets of analytical themes were identified. The first represents the disease trajectory and specific chronological points within it. The second details three sets of factors (internal, external and communicating) that impact on people's experiences of events along the disease trajectory. Analysing the intersections of these themes highlighted which palliative care needs are most required at each stage.

The findings highlighted the need for greater communication and information provision to allow timely, informed decision making, empowering individuals to maintain control of their care in face of the unremitting loss caused by the disease.

Conclusion Despite being a life-limiting condition people with MND and their carers have limited awareness of, or involvement with, palliative care services. There are clear points in the disease trajectory where palliative care input could enhance patient and carer experience of the disease, particularly at times of significant physical change.

\section{P-99 MULTI-DISCIPLINARY PALLIATIVE CARE FOR MEN LIVING WITH DUCHENNE: A QUALITATIVE INTERVIEW STUDY}

Sheonad Laidlaw, Emma Carduff. Marie Curie Care, Glasgow, UK

\subsection{6/bmjspcare-2018-hospiceabs. 124}

Background Duchenne Muscular Dystrophy (DMD) is an $\mathrm{x}$ linked, ultra-rare neuromuscular condition affecting 1 in 3600-6000 live male births (Bushby, Finkel, Birnkrant et al., 2010). Individuals live with an exceptional illness trajectory of prolonged dwindling, frailty and high symptom burden (Landfeldt, Lindgren, Bell et al., 2014). While it is recognised that a co-ordinated multidisciplinary team approach may increase the survival of those with DMD and improve their quality of life (QoL) (Bushby, Finkel, Birnkrant et al., 2010), adults are receiving less comprehensive and co-ordinated care compared with those in the paediatric service (Rodger, Woods, Bladen et al., 2015).

Aim To investigate QoL in adults with DMD living in the West of Scotland (WoS).

Methods The SEIQoL-DW tool was used to assess the five most important elements that contribute to an individual's QoL - these were then used to guide qualitative interviews with six men in the WoS. A thematic analysis was undertaken. Results Men living with DMD in the WoS described living good lives, but feel 'forgotten' due to perceived gaps in their care: poorly co-ordinated and infrequent health care; lack of multi-disciplinary team input and holistic care; and poor or no access to allied health care professionals, for example physiotherapy and psychological support.

Conclusions Existing guidelines rarely seem to materialise as person-centred care. There are numerous opportunities to introduce palliative care gently, as part of the MDT, early in the illness trajectory and continue in a dynamic manner as time elapses and when trigger points arise. Better co-ordinated multi-disciplinary care, with the inclusion of a palliative care specialist, may be a solution allowing for an early introduction to palliative care and proactive advance care planning.

\section{P-100 DELIVERING COLLABORATIVE EDUCATION TO ENHANCE PATIENT-CENTRED DECISION MAKING IN RENAL FAILURE}

'Sue Goodall, ${ }^{2}$ Pamela Dallyn, 'Suzanne Ford-Dunn. 'St Barnabas House Hospice, Worthing, UK; ${ }^{2}$ Sussex Kidney Unit, Brighton, UK

10.1136/bmjspcare-2018-hospiceabs. 125

Background There is increasing recognition that dialysis may not offer significant survival advantage or improvement of symptoms, in older patients with high co-morbidity (Hussain, Mooney \& Russon, 2013; Chandna, Da Silva-Gane, Marshall et al., 2011; Davidson \& Sheerin, 2013; Verberne, Geers, Jellema et al., 2016; Foote, Kotwal, Gallagher et al., 2016). Despite this the number of people over the age of 75 starting dialysis in the UK continues to increase (UK Renal Registry, 2017). Several studies have raised concern that patients are not fully informed about conservative management, or the risks and burdens associated with dialysis, (Brennan, Stewart, Burgess et al., 2017; Davidson, 2010) and around $60 \%$ of patients regret their decision to commence dialysis (Davidson, 2010; Muthalagappan, Johansson, Kong et al., 2013), many reporting it was the wish of their physician or family. Locally, patients with renal failure are invited to a patient/carer education session providing detailed information about the different types of dialysis on offer, and individual sessions to revisit options and introduce conservative management. The group session lacked detail on conservative management and the renal supportive service provided by the local hospice.

Aim To improve the decision-making process ensuring patients fully understand all available options, reducing inappropriate commencement of dialysis and patient regret.

Method The author met with the renal nurse manager who provides dialysis modality education in the local area, and offered to deliver information on conservative management and the service provided by the local hospice.

Results To date there have been three collaborative education sessions, providing information to 17 patients and carers. Evaluation of these sessions are consistently positive. Comments include; 'informative, brilliant, clear explanation of options, we did not know there were so many options, thank you for the information and help'. At the latest session attended by two patients over the age of 75 with multiple comorbidities and their carers, both patients chose conservative management over dialysis.

Conclusion The success of this project demonstrated that inter-organisational collaboration between the hospice renal service and the hospital renal service enhanced the delivery of information, promoting fully informed patient-centred decision making. 